\title{
Directed forgetting of actions by younger and older adults
}

\author{
JULIE L. EARLES \\ Honors College at Florida Atlantic University, Jupiter, Florida \\ and \\ ALAN W. KERSTEN \\ Florida Atlantic University, Boca Raton, Florida
}

\begin{abstract}
Memory for actions that are performed is substantially better than memory for descriptions of actions (e.g., Earles, 1996). In fact, people may form memories for actions even if they do not intend to or want to remember them. The directed forgetting paradigm was used to test the ability of younger and older adults to intentionally forget simple actions (also known as subject-performed tasks, or SPTs). Participants were asked to perform the action described by a verb-noun pair (e.g., break toothpick) or to read the pair, but not to perform the action. Following each pair, the participants were told either to remember or to forget the pair. Younger adults intentionally forgot verbally encoded pairs significantly better than did older adults. Actions that were performed, however, were difficult for both younger and older adults to intentionally forget. The performance of an action thus seems to result in strong item-specific processing that makes the action difficult to intentionally forget even for younger adults who can successfully intentionally forget verbally encoded items.
\end{abstract}

When people actively participate in an event, it may not only be easy for them to remember the event, but it may also be difficult for them to forget the event even if they intend to or want to forget it. Although younger adults may be better than older adults at intentionally forgetting verbally encoded information, both younger and older adults may have difficulty not remembering performed actions even if they try to forget them.

\section{Memory for Actions}

The actions involved in events seem to be especially easy for people to remember. Previous research has demonstrated that both younger and older adults recall simple performed actions (e.g., break stick, raise arm; often called subject performed tasks, or SPTs) better than verbal descriptions of actions (e.g., Bäckman \& Nilsson, 1985; Cohen, 1981; Earles, Kersten, Turner, \& McMullen, 1999). In fact, people seem to remember performed actions even if they do not intend to remember them. For example, Kausler and Hakami (1983) found that participants who were instructed to remember performed actions did not recall significantly more actions than did participants who were not told of the upcoming memory test. In other words, incidental learning of actions seems to be as good as intentional learning of actions.

The authors thank Noah Downie, Jennifer Godwin, and Meisha Merriweather for their assistance. Correspondence concerning this article should be addressed to J. L. Earles, Honors College, Florida Atlantic University, Jupiter, FL 33458 (e-mail: jearles@ fau.edu).
The encoding processes that take place during performance of an action may be different from those used to encode verbally presented items. Engelkamp (1998) suggests that performance of an action is an intentional act that results in self-awareness of performance. He suggests that when a person performs an action, this prevents the person from using other encoding strategies that are not directly connected with the enactment. Thus, strategies such as rehearsal and the ability to link the action with other meaningful information may be reduced or eliminated by performance.

Some evidence that encoding strategies, such as rehearsal, that are used for verbal learning are not used for remembering performed actions comes from studies on serial position curves produced during the recall of actions. A primacy effect usually occurs in the recall of lists of items because the first items on a list receive more processing, often in the form of rehearsal, than do later items. Unlike recall of verbally encoded items, such as words, recall of actions does not produce a large primacy effect (Cohen, 1981). This lack of a primacy effect for action memory suggests that people remember actions without using rehearsal (Cohen, 1984).

Linking new information with other meaningful information, such as contextualinformation, is also a useful strategy for encoding verbally presented information that does not seem to be used for remembering performed actions. Engelkamp and Dehn (2000) suggest that performance focuses attention on information relevant to performance and decreases attention to everything else, including the context. In support of this suggestion, they found that par- 
ticipants had difficulty remembering the presentation order of performed actions.

Hunt and Einstein (1981) distinguished between itemspecific processing, which emphasizes those features of an item that make the item different from other items, and relational processing, which emphasizes those features of an item that make it related to other items on a list. There is some empirical evidence that item-specific processing is increased by performance, whereas relational processing is decreased. For example, Olofsson (1997) examined memory for a list of action phrases that were either performed or not performed. Following presentation, participants were given three successive free-recall tests. Itemspecific processing should increase gains across successive tests, whereas relational processing should protect against losses across successive tests. The performed actions showed more gains across recall tests than did the nonperformed action phrases, which is evidence that performance increases item-specific processing. The performed actions also showed greater losses across lists, which is evidence that performance decreases relational processing. Thus, people do not seem to use the same strategies for remembering performed actions that they use for remembering verbally presented items.

Even when forced to use a strategy that is useful for remembering verbal items, this strategy use does not seem to enhance the recall of performed actions to the same extent as it enhances recall of nonperformed action phrases. For example,Zimmer and Engelkamp (1999) found that a conceptual orienting task enhanced memory for nonperformed action phrases more than for performed actions. Actions thus seem to be remembered without the use of intentional encoding strategies.

Participants may remember actions well without the use of additional memory strategies because performance guides the item-specific processing of an action. Memory for actions may thus be good because performance of an action results in good item-specific processing. When a verb is performed, this makes the verb become more specific (Earles \& Kersten, 2000). For example, when a person performs the action close eyes, the specific understanding of the verb close is distinguished from the meaning suggested by the action close door, and when a person performs the action wind clock, the specific understanding of wind is distinguished from the meaning suggested by wind string. Performance also increases the item-specific processing of the noun because either an object or an imagined object is used for performance. During performance, the participant thus forms an image of the object in combination with the verb.

This performance-guided encoding of item-specific information may be especially helpful for older adults. Older adults often have more difficulty than younger adults with memory tasks that involve strategic processing. As the need for self-initiated strategic processing decreases, age differences often decrease as well (Craik \& Jennings, 1992). Thus, if performance produces a strong memory trace by increasing item-specific information without the use of self-initiated encoding strategies, older adults may benefit from this guidance more than do younger adults.

\section{Directed Forgetting}

As was mentioned above, the performance of an action may result in item-specific processing that produces a memory trace that is so strong that people remember the action even if they do not want to remember it. They may, in fact, remember an action even if they actively try to forget it. Directed forgetting is one method that has been used to examine the ability to forget. In the directed forgetting paradigm, people are asked to remember some items and to forget other items (Bjork, 1970). In most circumstances, recall of words that are accompanied by an instruction to remember has been found to be better than recall of words that are accompanied by an instruction to forget (e.g., MacLeod, 1975; see MacLeod, 1998, for a review).

This directed forgetting effect does not seem to be due to participants' intentionally failing to report memory for words that they actually do remember. For example, when MacLeod (1999) offered to pay participants for each additional word that they could recall, the participants did not recall more of the words. This suggests that the directed forgetting effect is not due to demand characteristics.

There are two basic methods used to measure directed forgetting. Participants receive the instruction to remember or forget either following the presentation of each item (i.e., the item method of directed forgetting) or following presentation of blocks of items (i.e., the list method of directed forgetting). In the present study, the item method of directed forgetting was used. The better recall of remember than of forget items on the item method directed forgetting task appears to be due to selective processing of the remember items (Bjork, 1972). Participants seem to wait for the cue before they rehearse the items (MacLeod, 1999). They are then motivated to rehearse the remember items and discouraged from rehearsing the forget items (Basden, Basden, \& Gargano, 1993; MacLeod \& Daniels, 2000). Participants thus seem to separate the remember items out for item-specific processing (Basden \& Basden, 1998). Therefore, participants do not encode items that they are asked to forget as well as they encode items that they are asked to remember, and remember items are thus retrieved better during the recall task (Johnson, 1994).

Performance of an action may reduce the directed forgetting effect because performance increases item-specific processing. MacLeod and Daniels (2000) suggest that if an item receives elaborative item-specific, conceptual processing before the instruction to forget, the forget and the remember items are not encoded differently, and the directed forgetting effect should be reduced. In other words, they suggest that an increase in item-specific processing should decrease the directed forgetting effect. In support of this theory, they found that if participants generated words from definitions during study, the directed forgetting effect was eliminated. They suggest that this occurred because selective rehearsal could not override the strong conceptual encoding produced by generation. 
Thus, it follows from the theory of MacLeod and Daniels (2000) that if performance forces item-specific processing, both the remember and the forget items will have been encoded before the instruction is presented, and further selective rehearsal of the remember items will not increase memory for those items. Thus, performed actions should yield a smaller directed forgetting effect than should nonperformed action phrases, because participants have already encoded the action before the instruction to remember or forget is received.

\section{Age Differences in Directed Forgetting}

Older adults have been found to have more difficulty than younger adults intentionally forgetting verbally presented items (Zacks, Radvansky, \& Hasher, 1996). The directed forgetting effect may not be as large for older as for younger adults because older adults have difficulty suppressing the to-be-forgotten items and focusing on the tobe-remembered items. There is much evidence that older adults have difficulty preventing the processing of irrelevant information (e.g., Connelly, Hasher, \& Zacks, 1991; Earles, Connor, Frieske, Park, \& Smith, 1997; Hartman \& Hasher, 1991; Hasher, Stoltzfus, Zacks, \& Rypma, 1991). Thus, for older adults, to-be-forgotten information may enter working memory even though it is irrelevant to and may actually hinder memory for to-be-remembered items. Unlike younger adults, older adults may therefore process the to-be-forgotten items almost as well as the to-beremembered items, resulting in a reduction in the directed forgetting effect.

This age difference in directed forgetting is expected to be smaller when actions are performed. Thus, we expected a three-way interaction among age, encoding method (i.e., performed vs. nonperformed), and memory instruction (i.e., remember vs. forget). For nonperformed action phrases, younger adults were expected to selectively rehearse the remember items, whereas older adults were expected to treat forget items similarly to remember items, resulting in a larger directed forgetting effect for the younger adults. For performed actions, on the other hand, performance was expected to result in strong item-specific processing before the instruction to remember or forget, so forget items were expected to be recalled almost as well as remember items for both younger and older adults. In other words, performance of an action was expected to produce a strong memory trace that is difficult to suppress even for younger adults who can successfully intentionally suppress nonperformed action phrases.

\section{METHOD}

\section{Participants}

The participants were 54 undergraduate students, 18-22 years of age $(M=19.16, S D=1.04)$, and 54 community-dwelling older adults, $60-85$ years of age $(M=73.27, S D=6.41)$. The undergraduates received course credit for participation, and the older adults received $\$ 10$. The participants rated their health status on a scale of 1 (poor) to 5 (excellent). The younger adults had significantly higher health ratings $(M=4.34, S D=0.64)$, than did the older adults $[M=$ $3.98, S D=0.64 ; t(107)=2.93, p=.004]$.

\section{Materials}

There was one list of 40 verb-noun pairs. Each pair described a simple action (e.g., flip coin, nod head). Half of the pairs contained an external object (e.g., coin), and half did not (e.g., head). These pairs can be found in the Appendix.

\section{Design}

There were three independent variables, age (younger or older), encoding method (performed or not performed), and instruction (remember or forget). Type of instruction was manipulated within subjects, and age and encoding method were manipulated between subjects. The dependent variable was the proportion of verb-noun pairs recalled.

\section{Procedure}

There were 40 verb-noun pairs that described simple actions. Each pair was presented on an $8.5 \times 11$ in. card for $8 \mathrm{sec}$. Half of the participants performed the actions described by the pairs. The other half of the participants read, but did not perform, the action described by each pair.

Following the presentation of each action phrase, the participants were instructed to either remember or forget the preceding phrase. For the remember items, they were shown an $8.5 \times 11$ in. card containing the word remember, and for the forget items, they were shown a card containing the word forget. This card was shown for $6 \mathrm{sec}$. For half of the verb-noun pairs, the participants received the remember instruction, and for half of the pairs they received the forget instruction. A random order of instruction was chosen, with the constraint that the same instruction was not given for more than three pairs in a row. Half of the participants received the memory instructions in this order, and half received the instructions in the reverse order. Immediately following presentation of the last item, the participants were asked to recall all 40 of the verb-noun pairs. They received as much time as they needed to complete this task.

\section{RESULTS}

A 2 (encoding method, performed or not performed) $\times 2$ (memory instruction, remember or forget) $\times 2$ (age, younger or older) analysis of variance was conducted. The means are shown in Figure 1. The participants who performed the actions $(M=.50, S D=.18)$ recalled significantly more than those who did not $[M=.28, S D=.13 ; F(1,108)=$ $\left.101.81, M S_{\mathrm{e}}=0.028, p<.001\right]$. The participants recalled more of the verb-noun pairs that they were asked to remember $(M=.46, S D=.22)$ than of the pairs they were asked to forget $[M=.32, S D=.22 ; F(1,108)=71.85$, $\left.M S_{\mathrm{e}}=0.015, p<.001\right]$. Younger adults $(M=.49, S D=.17)$ recalled significantly more pairs than did older adults $\left[M=.29, S D=.16 ; F(1,108)=80.41, M S_{\mathrm{e}}=0.028, p<.001\right]$.

It was expected that older adults would show little effect of directed forgetting for both performed actions and nonperformed action phrases. Younger adults, on the other hand, were expected to show a greater effect of directed forgetting on memory for nonperformed action phrases than on memory for performed actions. Consistent with these predictions, there was a significant interaction of age, encoding method, and memory instruction $[F(1,108)=$ $\left.14.31, M S_{\mathrm{e}}=0.015, p<.001\right]$. This interaction is shown in Figure 1.

As can be seen in Figure 1, for the older adults, there was no significant interaction of encoding method and instruction $[F(1,54)<1]$. For the younger adults, on the 


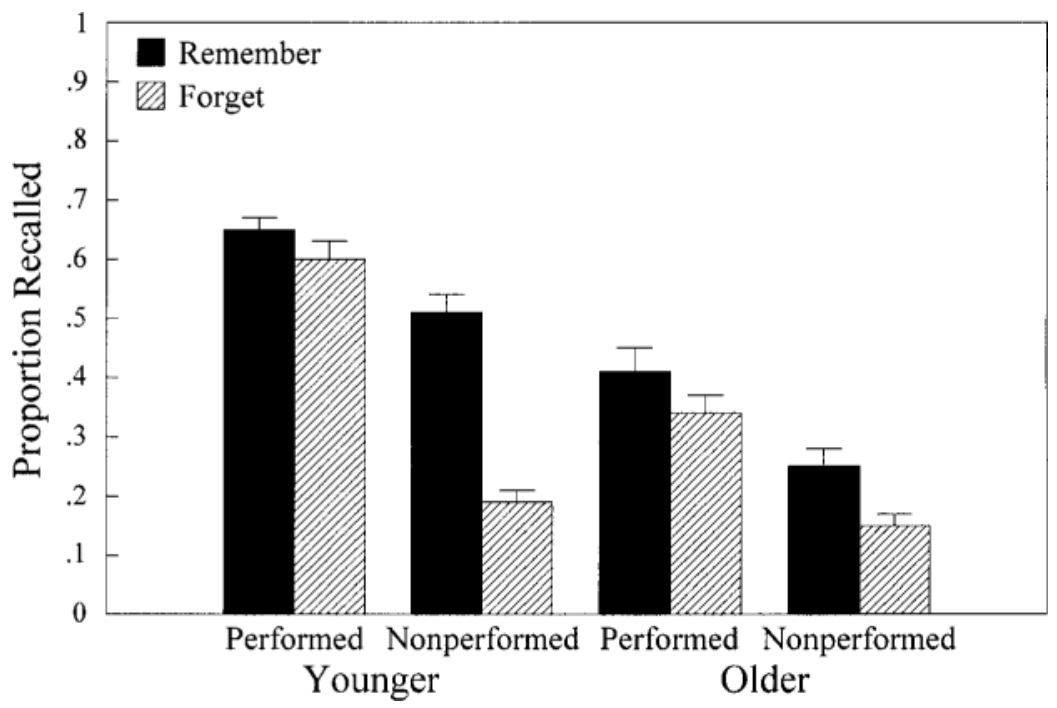

Figure 1. Interaction of encoding method, memory instruction, and age. Bars represent standard errors.

other hand, there was a significant interaction of encoding method and memory instruction $\left[F(1,54)=31.51, M S_{\mathrm{e}}=\right.$ $0.016, p<.001]$. For the performed actions, younger adults did not recall significantly more of the actions they were told to remember than of the actions they were told to forget $[t(27)=1.71, p=.10]$. For the nonperformed action phrases, younger adults recalled significantly more of the to-be-remembered phrases than of the to-be-forgotten phrases $[t(27)=8.64, p<.001]$. For younger adults, the directed forgetting effect was thus larger for the nonperformed action phrases than for the performed actions.

An additional measure of intentional forgetting ability can be provided by dividing the number of to-be-forgotten items recalled by each participant by the number of tobe-remembered items recalled. A person with perfect intentional forgetting ability would produce a score of zero, whereas a person with no intentional forgetting ability would produce a score of one. For performed items, the average ratio for younger adults was $.95(S D=.30)$ and for older adults $.92[S D=.46 ; t(54)<1]$. Thus, neither age group showed much intentional forgetting of performed items. For nonperformed action phrases, the average ratio for younger adults was $.42(S D=.24)$, and the average ratio for older adults was .63 [SD $=.45 ; t(52)=2.204, p=$ $.032] .{ }^{1}$ Thus, younger adults were better able to intentionally forget nonperformed action phrases than were older adults.

\section{DISCUSSION}

Performed actions were found to be more difficult to forget than were nonperformed action phrases. Because both younger and older adults found it difficult to intentionally forget performed actions, the age difference in directed forgetting that was found for verbally presented items was not found for performed actions.
This difficulty in forgetting actions may have both positive and negative practical implications. Memory for actions is very important for everyday functioning, and thus both younger and older adults are concerned about forgetting actions. Our research suggests that simple everyday actions are easier to remember and more difficult to forget than verbally presented information, which is good news for people worried about memory for actions. On the other hand, there are many events, such as traumatic events, that people may want to forget but find that they cannot forget. Our research suggests that once an action is performed, that action is difficult to forget even if someone wants to and tries to forget it.

In the item method of directed forgetting that was used in the present study, the directed forgetting effect is assumed to occur because the remember items are separated from the forget items and these remember items receive postperceptual processing in the form of selective rehearsal (MacLeod \& Daniels, 2000). It was hypothesized that an increase in item-specific processing should decrease the directed forgetting effect because it decreases the benefit of additional processing following the instruction to remember.

One reason that actions may be particularly easy to remember and difficult to forget is that when a person performs an action, this performance results in good itemspecific processing. The person's attention is focused on the action. When an action is performed, participants seem to be unable to wait for the memory instruction before fully processing an action. They have already formed a strong memory trace, so additional processing following the instruction to remember does not greatly increase memory. In other words, selective rehearsal of some actions and not others does not seem to further enhance memory for those actions to the same extent that selective rehearsal benefits memory for nonperformed action phrases. Thus, additional 
encoding strategies do not seem to be needed in order for actions to be remembered. This finding is consistent with the finding of Zimmer and Engelkamp (1999) that traditional levels-of-processing manipulations are not as effective with performed actions as they are for verbally presented information. Not only are memory strategies that are effective for remembering verbally presented material not needed as much for memory for actions, our studies show that the memory trace for an action is formed even if the person actively tries not to remember the action.

Although the directed forgetting effect was greatly reduced for actions, there was a small directed forgetting effect. This finding of a very small effect of directed forgetting on memory for actions supports Engelkamp's (1998) theory that the encoding of actions contains both an automatic and a controlled component. The reason to-beremembered actions are recalled somewhat better than tobe-forgotten actions may be that there is increased memory for the verbal component of the action owing to selective rehearsal of the action phrase.

Good item-specific processing may occur during performance not only because performance enhances attention to the phrase, but also because performance enhances the interpretation of the action phrase. Performance does more than just repeat the action phrase. Performance specifies the meaning of the verb in the phrase and provides a concrete example of the noun. It thus provides additional information to the participant.

Performance is, in some ways, similar to generating a word from a definition, as was done by the participants in the study by Macleod and Daniels (2000). The action is defined, but the participant must interpret the definition and decide how to represent the action. An action must therefore be generated from the action phrase. The results of the present study are thus consistent with those of MacLeod and Daniels, who found a decreased directed forgetting effect when words were generated from definitions.

Performance of an action is also similar in some ways to forming an image. Producing an image is one method of increasing item-specific processing, so following the theory of MacLeod and Daniels (2000), one would expect that forming an image would decrease the directed forgetting effect, just as we found a decrease in the directed forgetting effect for actions. Basden and Basden (1998), however, found a larger directed forgetting effect for pictures than for words.

The most likely explanation for the difference between our results and those of Basden and Basden (1998) is that they used categorizable lists of words. Golding, Long, and MacLeod (1994) found that when words are related to each other, it is difficult to separate the remember items from the forget items, and thus the directed forgetting effect is reduced. Basden and Basden suggested that when the words were verbally presented, the participants related the words to each other by clustering items into categories, and thus the directed forgetting effect was reduced because the to-be-remembered words were linked with the to-beforgotten words. When pictures were presented, on the other hand, they suggested that this increased the item-specific processing of the items, making it more difficult to conduct relational processing. Basden and Basden thus suggested that the directed forgetting effect was larger for pictures than for words because the to-be-remembered pictures and the to-be-forgotten pictures were not placed into categories but the words were categorized.

The relatedness of the action pairs was not manipulated in the present study. However, if the actions were related to each other, such as the actions in a complex event, like baking a cake, this would be expected to reduce the directed forgetting effect even further. Thus, in everyday events in which there is a series of related actions, it is expected that the actions would be even more difficult to forget than were the unrelated actions in the present study.

Because both younger and older adults had difficulty forgetting the performed actions, there was no significant age difference in the directed forgetting of performed actions. As was found by Zacks et al. (1996), older adults did show some directed forgetting of verbally encoded items, but the ability to limit the processing of information that they did not want to or intend to process was reduced, as compared with that of younger adults. Thus, for nonperformed action phrases, younger adults showed a larger effect of directed forgetting than did older adults. As was suggested by Zacks et al. and others (e.g., Earles et al., 1997), older adults may have difficulty choosing not to remember irrelevant information. Older adults may have difficulty inhibiting the processing of the to-be-forgotten verb-noun pairs and thus may process the to-be-forgotten pairs almost as well as the to-be-remembered pairs.

\section{CONCLUSIONS}

The results of this experiment demonstrate that although younger adults are better than older adults at intentionally forgetting verbally encoded information, both younger and older adults have difficulty intentionally forgetting performed actions. Performance seems to guide the itemspecific processing of an action, making the action easy to remember even if the person does not intend to or want to remember it.

\section{REFERENCES}

BÄckman, L., \& Nilsson, L.-G. (1985). Prerequisites for lack of age differences in memory performance. Experimental Aging Research, 11, 67-73.

BASDEn, B. H., \& BASDEN, D. R. (1998). Directed forgetting: A contrast of methods and interpretations. In J. M. Golding \& C. M. MacLeod (Eds.), Intentionalforgetting: Interdisciplinary approaches (pp. 139172). Mahwah, NJ: Erlbaum.

Basden, B. H., Basden, D. R., \& Gargano, G. J. (1993). Directed forgetting in implicit and explicit memory tests: A comparison of methods. Journal of Experimental Psychology: Learning, Memory, \& Cognition, 19, 603-616.

BJork, R. A. (1970). Positive forgetting: The noninterference of items intentionally forgotten. Journal of Verbal Learning \& Verbal Behavior, 9, 255-268.

BJork, R. A. (1972). Theoretical implications of directed forgetting. In A. W. Melton \& E. Martin (Eds.), Coding processes in human memory (pp. 217-235). Washington, DC: Winston.

Cohen, R. L. (1981). On the generality of some memory laws. Scandanavian Journal of Psychology, 22, 267-281. 
Cohen, R. L. (1984). Individual differences in event memory: A case for nonstrategic factors. Memory \& Cognition, 12, 633-641.

Connelly, S. L., Hasher, L., \& Zacks, R. T. (1991). Age and reading: The impact of distraction. Psychology \& Aging, 6, 533-541.

Craik, F. I. M., \& Jennings, J. M. (1992). Human memory. In F. I. M. Craik \& T. A. Salthouse (Eds.), The handbook of aging and cognition (pp. 51-110). Hillsdale, NJ: Erlbaum.

EARLES, J. L. (1996). Adult age differences in recall of performed and nonperformed items. Psychology \& Aging, 11, 638-648.

Earles, J. L., Connor, L. T., Frieske, D., Park, D. C., \& Smith, A. D. (1997). Age differences in inhibition: Possible causes and consequences. Aging, Neuropsychology, \& Cognition, 4, 45-57.

EARLES, J. L., \& Kersten, A. W. (2000). Adult age differences in memory for verbs and nouns. Aging, Neuropsychology, \& Cognition, 7, 130-139.

Earles, J. L., Kersten, A. W., Turner, J. M., \& McMullen, J. (1999). Influences of age, performance, and item-relatedness on verbatim and gist recall of verb-noun pairs. Journal of General Psychology, 126, 97 110.

Engelkamp, J. (1998). Memory for actions. East Sussex, U.K.: Psychology Press.

ENGELKAMP, J., \& DEHN, D. M. (2000). Item and order information in subject-performed tasks and experimenter-performed tasks. Journal of Experimental Psychology: Learning, Memory, \& Cognition, 26, 671-682.

Golding, J. M., Long, D. L., \& MacLeod, C. M. (1994). You can't always forget what you want: Directed forgetting of related words. Journal of Memory \& Language, 33, 493-510.

Hartman, M., \& Hasher, L. (1991). Aging and suppression: Memory for previously relevant information. Psychology \& Aging, 6, 587-594.

Hasher, L., Stoltzfus, E. R, Zacks, R. T., \& Rypma, B. (1991). Age and inhibition. Journal of Experimental Psychology: Learning, Memory, \& Cognition, 17, 163-169.

Hunt, R. R., \& Einstein, G. O. (1981). Relational and item-specific in- formation in memory. Journal of Verbal Learning \& Verbal Behavior, 20, 497-514.

JoHnson, H. M. (1994). Processes of successful intentional forgetting. Psychological Bulletin, 116, 274-292.

KAUSLER, D. H., \& HAKAmI, M. K. (1983). Memory for activities: Adult age differences and intentionality.Developmental Psychology, 19, 889894.

MacLeod, C. M. (1975). Long-term recognition and recall following directed forgetting. Journal of Experimental Psychology: Human Learning \& Memory, 1, 271-279.

MacLeod, C. M. (1998). Directed forgetting. In J. M. Golding \& C. M. MacLeod (Eds.), Intentionalforgetting: Interdisciplinaryapproaches (pp. 1-57). Mahwah, NJ: Erlbaum.

MacLeod, C. M. (1999). The item and list methods of directed forgetting: Test differences and the role of demand characteristics. Psychonomic Bulletin \& Review, 6, 123-129.

MacLeod, C. M., \& Daniels, K. A. (2000). Direct versus indirect tests of memory: Directed forgetting meets the generation effect. Psychonomic Bulletin \& Review, 7, 354-359.

Olofsson, U. (1997). Win some, lose some: Hypermnesia for actions reflects increased item-specific processing. Memory \& Cognition, 25, 797 800.

Zacks, R. T., RAdVAnsky, G., \& HASher, L. (1996). Studies of directed forgetting in older adults. Journal of Experimental Psychology: Learning, Memory, \& Cognition, 22, 143-156.

Zimmer, H. D., \& ENGelKamp, J. (1999). Levels-of-processing effects in subject-performed tasks. Memory \& Cognition, 27, 907-914.

\section{NOTE}

1. Two older adults were not included in this analysis because they recalled no items in one condition.

\begin{tabular}{ll} 
APPENDIX & $\begin{array}{c}\text { APrb-Noun Pairs } \\
\text { Verb-Nip coin }\end{array}$ \\
\hline squeeze eraser & stomp feet \\
pucker lips & shake egg \\
polish fork & nod head \\
rub stomach & fold napkin \\
draw circle & shrug shoulders \\
puff cheeks & cut fabric \\
break toothpick & cross fingers \\
flex muscles & smell book \\
drop bean & raise eyebrows \\
clench teeth & twist wire \\
tear paper & beat chest \\
pinch nose & mash clay \\
pat flower & wiggle toes \\
lick thumb & punch bag \\
count rocks & scratch ear \\
tickle elbow & tie knot \\
stretch balloon & wave hand \\
touch knee & hold ball \\
caress feather & bat eyelids \\
bite arm &
\end{tabular}

(Manuscript received February 8, 2000;

revision accepted for publication July 2, 2001.) 